\title{
Existence of Periodic Solutions to Multidelay Functional Differential Equations of Second Order
}

\author{
Cemil Tunç and Ramazan Yazgan \\ Department of Mathematics, Faculty of Sciences, Yüzüncü Yıl University, 65080 Van, Turkey \\ Correspondence should be addressed to Cemil Tunç; cemtunc@yahoo.com
}

Received 23 August 2013; Accepted 3 October 2013

Academic Editor: S. A. Mohiuddine

Copyright (C) 2013 C. Tunç and R. Yazgan. This is an open access article distributed under the Creative Commons Attribution License, which permits unrestricted use, distribution, and reproduction in any medium, provided the original work is properly cited.

Using Lyapunov-Krasovskii functional approach, we establish a new result to guarantee the existence of periodic solutions of a certain multidelay nonlinear functional differential equation of second order. By this work, we extend and improve some earlier result in the literature.

\section{Introduction}

It is well known that the problem of the existence of periodic solutions of retarded functional differential equations of second order is not only very important in the background applications, but also of considerable significance in theory of differential equations. Besides, the scope of retarded functional differential equations is very general. For example, it contains ordinary differential equations, differentialdifference equations, integrodifferential equations, and so on. The motivation of this paper is that in recent years the study of the existence of periodic solutions to various kinds of retarded functional differential equations of second order has become one of the most attractive topics in the literature. Especially, by using the famous continuation theorem of degree theory (see Gaines and Mawhin [1]), many authors have made a lot of interesting contributions to the topic for retarded functional differential equations of second order. Here, we would not like to give the details of these works.

On the other hand, amongst the achieved excellent results, one of them is the famous Yoshizawa's theorem [2] for existence of periodic solutions of retarded functional differential equations, which has vital influence and has been widely used in the literature. This theorem has also been generally one of the best results in the literature from the past till now. It should be noted that in 1994, Zhao et al. [3] proved four sufficiency theorems on the existence of periodic solutions for a class of retarded functional differentials to have the existence of an $\omega$-periodic solution. By this work, the authors proved that their theorems are better than Yoshizawa's [2] periodic solutions theorem primarily by removing restrictions of the size of the constant delay $h$. An example of application was given at the end of the paper. Namely, in the same paper, the authors applied the following Theorem A to discuss the existence of an $\omega$-periodic solution of the nonlinear delay differential equation of the second order:

$$
x^{\prime \prime}(t)+a x^{\prime}(t)+g(x(t-\tau))=p(t),
$$

where $p(t)$ is an external force, $g(x(t-\tau))$ is a delayed restoring force, the delay $\tau$ is a positive constant and the friction is proportional to the velocity, and $a$ is a positive constant. It should be noted that a feedback system with friction proportional to velocity, an external force $p(t)$, and a delayed restoring force $g(x(t-\tau)),(\tau>0)$ may be written as (1) (see Burton [4]).

Consider the following general nonautonomous delay differential equation:

$$
\begin{array}{r}
\dot{x}=F\left(t, x_{t}\right), \quad x_{t}=x(t+\theta), \\
-h \leq \theta \leq 0, t \geq 0,
\end{array}
$$

where $F: \Re \times C \rightarrow \mathfrak{R}^{n}, C=C\left([-h, 0], \mathfrak{R}^{n}\right), h$ is a positive constant, and we suppose that $F$ is continuous, $\omega$-periodic, and takes closed bounded sets into bounded sets of $\Re^{n}$; and such that solutions of initial value problems are unique, $h$ 
can be either larger than $\omega$, or equal to or smaller than $\omega$. Here $(C,\|\cdot\|)$ is the Banach space of continuous function $\phi$ : $[-h, 0] \rightarrow \Re^{n}$ with supremum norm; $h>0, C_{H}$ is the open $H$-ball in $C ; C_{H}:=\left\{\phi \in C\left([-h, 0], \mathfrak{R}^{n}\right):\|\phi\|<H\right\}$. Standard existence theory, see Burton [4], shows that if $\phi \in C_{H}$ and $t \geq 0$, then there is at least one continuous solution $x\left(t, t_{0}, \phi\right)$ such that on $\left[t_{0}, t_{0}+\alpha\right)$ satisfying (2) for $t>t_{0}, x_{t}(t, \phi)=\phi$ and $\alpha$ is a positive constant. If there is a closed subset $B \subset C_{H}$ such that the solution remains in $B$, then $\alpha=\infty$. Further, the symbol $|\cdot|$ will denote a convenient norm in $\mathfrak{R}^{n}$ with $|x|=$ $\max _{1 \leq i \leq n}\left|x_{i}\right|$. Let us assume that $C(t)=\left\{\phi:[t-\alpha, t] \rightarrow \mathfrak{R}^{n} \mid\right.$ $\phi$ is continuous $\}$ and $\phi_{t}$ denotes the $\phi$ in the particular $C(t)$, and that $\left\|\phi_{t}\right\|=\max _{t-\alpha \leq s \leq t}|\phi(t)|$. Finally, by the periodicity, we mean that that there is an $\omega>0$ such that $F(t, \phi)$ is $\omega$ periodic in the sense that if $x(t)$ is a solution of (2) so is $x(t+\omega)$.

Definition 1. Solutions of (2) are uniform bounded at $t=0$ if for each $B_{1}$ there exists $B_{2}$ such that $\left[\phi \in C,\|\phi\|<B_{1}, t \geq 0\right]$ imply that $|x(t, 0, \phi)|<B_{2}$ (see Burton [4]).

Definition 2. Solutions of (2) are uniform ultimate bounded for bound $B$ at $t=0$ if for each $B_{3}>0$ there exists a $K>0$ such that $\left[\phi \in C,\|\phi\|<B_{3}, t \geq K\right]$ imply that $|x(t, 0, \phi)|<B$ (see Burton [4]).

The first theorem given in Zhao et al. [3] is the following.

Theorem A. If the solutions of (2) are ultimately bounded by the bound $B$, then

(i) equation (2) has an w-periodic solution and is bounded by $B$,

(ii) if (2) is autonomous, then (2) has an equilibrium solution and is bounded by B (see Zhao et al. [3]).

Regarding (1) Zhao et al. [3] proved the following theorem as example of application.

Theorem B. Assume that the following conditions hold:

(1) $p(t)$ is an w-periodic continuous function, $g$ is a continuous differentiable function,

(2) $\lim _{|x| \rightarrow \infty} g(x) \operatorname{sgn} x=\infty$, and there is a bounded set $\Omega$ containing the origin such that $\left|g^{\prime}(x)\right| \leq c$ on $\Omega^{c} ; \Omega^{c}$ is the complement of the set $\Omega$,

(3) $\tau c<a$.

Then, (1) has an w-periodic motion.

Moreover, when $p(t)=K, K$ is a constant, under the above conditions (1) has a constant motion $x=c_{0}$, and the constant $c_{0}$ satisfies $g\left(c_{0}\right)=K$. In fact, from the condition (2), there is a bounded set $\Omega_{1}$ containing the origin such that $\left|g^{\prime}(x)\right| \leq c$ and $\int_{0}^{x} g(s) d s>0$ on $\Omega_{1}^{c} ; \Omega_{1}^{c}$ is the complement of the set $\Omega_{1}$.
In this paper, we consider the following nonlinear differential equation of second order with multiple constant delays, $\tau_{i}(>0)$ :

$$
\begin{aligned}
& x^{\prime \prime}(t)+\left\{f\left(x(t), x^{\prime}(t)\right)+g\left(x(t), x^{\prime}(t)\right) x^{\prime}(t)\right\} x^{\prime}(t) \\
& +h(x(t))+\sum_{i=1}^{n} g_{i}\left(x\left(t-\tau_{i}\right)\right)=p(t),
\end{aligned}
$$

where $\tau_{i}$ are fixed constants delay with $t-\tau_{i} \geq 0$; the primes in (3) denote differentiation with respect to $t \in \mathfrak{R}, f, g, h, g_{i}$, and $p$ are continuous functions in their respective arguments on $\mathfrak{R}^{2}, \mathfrak{R}^{2}, \mathfrak{R}, \mathfrak{R}$, and $\mathfrak{R}$, respectively, and also depend only on the arguments displayed explicitly. The continuity of these functions is a sufficient condition for existence of the solution of (3). It is also assumed as basic that the functions $f, g, h$, and $g_{i}$ satisfy a Lipschitz condition in $x, x^{\prime}, x\left(t-\tau_{1}\right), x(t-$ $\left.\tau_{2}\right), \ldots, x\left(t-\tau_{n}\right)$. By this assumption, the uniqueness of solutions of (3) is guaranteed. The derivatives $d g_{i} / d x \equiv g_{i}^{\prime}(x)$ exist and are continuous. It should be noted that throughout the paper, sometimes, $x(t)$ and $y(t)$ are abbreviated as $x$ and $y$, respectively.

We write (3) in system form as follows:

$$
\begin{gathered}
x^{\prime}=y, \\
y^{\prime}=-\{f(x, y)+g(x, y) y\} y-h(x)-\sum_{i=1}^{n} g_{i}(x) \\
+\sum_{i=1}^{n} \int_{-\tau_{i}}^{0} g_{i}^{\prime}(x(t+s)) y(t+s) d s+p(t),
\end{gathered}
$$

where $g_{i}^{\prime}(x(t+s))=d g_{i} / d x$.

It is clear that (3) is a particular case of (2). It should be noted that the reason or the motivation for taking into consideration (3) comes from the following modified Liénard type equation of the form:

$$
x^{\prime \prime}(t)+\left\{f(x(t))+g(x(t)) x^{\prime}(t)\right\} x^{\prime}(t)+h(x(t))=e(t) .
$$

These types of equations have great applications in theory and applications of the differential equations. Therefore, till now, the qualitative behaviors, the stability, boundedness, global existence, existence of periodic solutions, and so forth, of these type differential equations have been studied by many researchers, and the researches on these topics are still being done in the literature. For example, we refer the readers to the books of Ahmad and Rao [5], Burton [4], Gaines and Mawhin [1], and the papers of Constantin [6], Graef [7], Huang and Yu [8], Jin [9], Liu and Huang [10], Nápoles Valdés [11], Qian [12], Tunç [13-21], C. Tunç and E. Tunç [22], Zhao et al. [3], Zhou [23], and the references cited in these works.

We here give certain sufficient conditions to guarantee the existence of an $\omega$-periodic solution of (3). This paper is inspired by the mentioned papers and that in the literature. Our aim is to generalize and improve the application given in [3] for (3). This paper has also a contribution to the investigation of the qualitative behaviors of retarded functional 
differential equations of second order, and it may be useful for researchers who work on the above mentioned topics. Finally, without using the famous continuation theorem of degree theory, which belongs to Gaines and Mawhin [1], we prove the following main result. This case makes the topic of this paper interesting.

\section{Main Result}

Our main result is the following.

Theorem 3. We assume that there are positive constants $a, \bar{a}$, $b, \tau$, and $c_{i}$ such that the following conditions hold:

(i) $\bar{a} \geq f(x, y)+g(x, y) y \geq a$,

(ii) $\lim _{|x| \rightarrow \infty} h(x) \operatorname{sgn} x=\infty$, and there is a bounded set $\Omega$ containing the origin such that $\left|h^{\prime}(x)\right| \leq$ bon $\Omega^{c} ; \Omega^{c}$ is the complement of the set $\Omega$,

(iii) $\lim _{|x| \rightarrow \infty} g_{i}(x) \operatorname{sgn} x=\infty$, and there is a bounded set $\Omega$ containing the origin such that $\left|g_{i}^{\prime}(x)\right| \leq c_{i}$ on $\Omega^{c} ; \Omega^{c}$ is the complement of the set $\Omega$,

(iv) $p(t)$ is an w-periodic continuous function.

If $\tau \sum_{i=1}^{n} c_{i}<a$, then (3) has an $\omega$-periodic solution.

Moreover, $p(t)=K, K$-constant, under the above conditions (3) has a constant motion $x=c_{0}$, and the constant $c_{0}$ satisfies $h\left(c_{0}\right)+g_{i}\left(c_{0}\right)=K$.

Proof. Define the Lyapunov-Krasovskii functional $V=$ $V\left(x_{t}, y_{t}\right)$ :

$$
\begin{aligned}
V= & \frac{1}{2} y^{2}+\int_{0}^{x} h(s) d s+\sum_{i=1}^{n} \int_{0}^{x} g_{i}(s) d s \\
& +\sum_{i=1}^{n} \lambda_{i} \int_{-\tau_{i}}^{0} \int_{t+s}^{t} y^{2}(\theta) d \theta d s,
\end{aligned}
$$

where $\lambda_{i}$ are some positive constants to be determined later in the proof.

Evaluating the time derivative of $V$ along system (4), we get

$$
\begin{aligned}
\dot{V}= & -\{f(x, y)+g(x, y) y\} y^{2} \\
& +y \sum_{i=1}^{n} \int_{-\tau_{i}}^{0} g_{i}^{\prime}(x(t+s)) y(t+s) d s \\
& +y p(t)+\sum_{i=1}^{n} \lambda_{i} \int_{-\tau_{i}}^{0}\left(y^{2}(t)-y^{2}(t+s)\right) d s \\
= & -\{f(x, y)+g(x, y) y\} y^{2} \\
& +y \sum_{i=1}^{n} \int_{-\tau_{i}}^{0} g_{i}^{\prime}(x(t+s)) y(t+s) d s
\end{aligned}
$$

$$
\begin{aligned}
& +y p(t)+\sum_{i=1}^{n}\left(\lambda_{i} \tau_{i}\right) y^{2} \\
& -\sum_{i=1}^{n} \lambda_{i} \int_{-\tau_{i}}^{0} y^{2}(t+s) d s .
\end{aligned}
$$

By noting the assumption $\left|g_{i}^{\prime}(x)\right| \leq c_{i}$ of the theorem and the estimate $2|\alpha \gamma| \leq \alpha^{2}+\gamma^{2}$, one can obtain the following estimates:

$$
\begin{aligned}
y \sum_{i=1}^{n} \int_{-\tau_{i}}^{0} g_{i}^{\prime}(x(t+s)) y(t+s) d s \\
\quad \leq \sum_{i=1}^{n} \int_{-\tau_{i}}^{0}\left|g_{i}^{\prime}(x(t+s))\right||y(t)||y(t+s)| d s \\
\quad \leq \frac{1}{2} \sum_{i=1}^{n} \int_{-\tau_{i}}^{0} c_{i}\left(y^{2}(t)+y^{2}(t+s)\right) d s \\
\quad \leq \frac{1}{2} \sum_{i=1}^{n}\left(c_{i} \tau_{i}\right) y^{2}+\frac{1}{2} \sum_{i=1}^{n} c_{i} \int_{-\tau_{i}}^{0} y^{2}(t+s) d s .
\end{aligned}
$$

Then, it follows that

$$
\begin{aligned}
\dot{V} \leq & -\{f(x, y)+g(x, y) y\} y^{2}+y p(t) \\
& +\frac{1}{2} \sum_{i=1}^{n}\left(c_{i}+2 \lambda_{i}\right) \tau_{i} y^{2} \\
& +\frac{1}{2} \sum_{i=1}^{n}\left(c_{i}-2 \lambda_{i}\right) \int_{-\tau_{i}}^{0} y^{2}(t+s) d s .
\end{aligned}
$$

Let $\lambda_{i}=c_{i} / 2$ and $\tau=\max \left\{\tau_{1}, \ldots, \tau_{n}\right\}$. In fact, these choices imply that

$$
\begin{aligned}
\dot{V} & \leq-\left\{f(x, y)+g(x, y) y-\tau \sum_{i=1}^{n} c_{i}-|p(t)||y|^{-1}\right\} y^{2} \\
& \leq-\left(a-\tau \sum_{i=1}^{n} c_{i}-|p(t)||y|^{-1}\right) y^{2} .
\end{aligned}
$$

In view of the continuity and periodicity of the function $p$ and the assumption $a-\tau \sum_{i=1}^{n} c_{i}>0$, it follows that there is a bounded set $\Omega_{2} \supseteq \Omega_{1}$ with $\Omega_{2}$ containing the origin and a positive constant $\mu$ such that

$$
\mu \leq a-c \tau-\frac{|p(t)|}{|y|} \quad \text { for } \Re \times \Omega_{2}^{c} .
$$

Therefore, we can write

$$
\dot{V} \leq-\mu y^{2} \quad \text { for }(t, x, y) \in \Re \times \Omega_{2}^{c} \times \Omega_{2}^{c} .
$$

From the last estimate, we can arrive that the $y$-coordinate of the solutions of system (4) is ultimately bounded 
for a positive constant $\beta$. On the other hand, since $p$ is a continuous periodic function, if $|y| \leq \beta$ and $V_{1}=V+y$, then, subject to the assumptions of the theorem, it can be easily seen that there is a constant $K_{1}>0$ on $\Re \times \Omega_{2}^{c}$ such that

$$
\begin{aligned}
\dot{V}_{1}= & \dot{V}+y^{\prime} \\
= & -\{f(x, y)+g(x, y) y\} y^{2} \\
& +y \sum_{i=1}^{n} \int_{-\tau_{i}}^{0} g_{i}^{\prime}(x(t+s)) y(t+s) d s+y p(t) \\
& +\sum_{i=1}^{n}\left(\lambda_{i} \tau_{i}\right) y^{2}-\sum_{i=1}^{n} \lambda_{i} \int_{-\tau_{i}}^{0} y^{2}(t+s) d s \\
& -\{f(x, y)+g(x, y) y\} y-h(x)-\sum_{i=1}^{n} g_{i}(x) \\
& +\sum_{i=1}^{n} \int_{-\tau_{i}}^{0} g_{i}^{\prime}(x(t+s)) y(t+s) d s+p(t) \\
\leq & -a y^{2}+y \sum_{i=1}^{n} \int_{-\tau_{i}}^{0} g_{i}^{\prime}(x(t+s)) y(t+s) d s \\
\leq & -h(x)-\sum_{i=1}^{n} g_{i}(x)+K_{-\tau_{i}} \cdot g_{i}^{\prime}(x(t+s)) y(t+s) d s+p(t) \\
& +y p(t)+\sum_{i=1}^{n}\left(\lambda_{i} \tau_{i}\right) y^{2} \\
& -\sum_{i=1}^{n} \lambda_{i} \int_{-\tau_{i}}^{0} y^{2}(t+s) d s+\bar{a}|y|-h(x)-\sum_{i=1}^{n} g_{i}(x) \\
& +y^{0}(t)
\end{aligned}
$$

Since $h(x) \rightarrow \infty$ and $g_{i}(x) \rightarrow \infty$ as $x \rightarrow \infty$, then it can be chosen a positive constant $B_{1}$ such that

$$
\dot{V}_{1} \leq-0.5 \text { for } x \geq B_{1} \text {. }
$$

Therefore, we can conclude that there is a positive constant $\alpha_{1}$ such that the $x$-coordinate of the solutions of system (4) satisfies $x \leq \alpha_{1}$ for $|y| \leq \beta$.

In a similar manner, if $|y| \leq \beta$ and $V_{2}=V-y$, then, subject to the assumptions of the theorem, it can be easily followed by the time derivative of the functional $V_{2}$ that there is a constant $K_{2}>0$ on $\Re \times \Omega_{2}^{c}$ such that

$$
\begin{aligned}
\dot{V}_{2}= & \dot{V}-y^{\prime} \\
= & -\{f(x, y)+g(x, y) y\} y^{2} \\
& +y \sum_{i=1}^{n} \int_{-\tau_{i}}^{0} g_{i}^{\prime}(x(t+s)) y(t+s) d s+y p(t)
\end{aligned}
$$

$$
\begin{aligned}
& +\sum_{i=1}^{n}\left(\lambda_{i} \tau_{i}\right) y^{2}-\sum_{i=1}^{n} \lambda_{i} \int_{-\tau_{i}}^{0} y^{2}(t+s) d s \\
& +\{f(x, y)+g(x, y) y\} y+h(x)+\sum_{i=1}^{n} g_{i}(x) \\
& -\sum_{i=1}^{n} \int_{-\tau_{i}}^{0} g_{i}^{\prime}(x(t+s)) y(t+s) d s-p(t) \\
& \leq-a y^{2}+y \sum_{i=1}^{n} \int_{-\tau_{i}}^{0} g_{i}^{\prime}(x(t+s)) y(t+s) d s \\
& +y p(t)+\sum_{i=1}^{n}\left(\lambda_{i} \tau_{i}\right) y^{2}-\sum_{i=1}^{n} \lambda_{i} \int_{-\tau_{i}}^{0} y^{2}(t+s) d s \\
& +\bar{a}|y|+h(x)+\sum_{i=1}^{n} g_{i}(x) \\
& -\sum_{i=1}^{n} \int_{-\tau_{i}}^{0} g_{i}^{\prime}(x(t+s)) y(t+s) d s-p(t) \\
& \leq h(x)+\sum_{i=1}^{n} g_{i}(x)+K_{2} \text {. }
\end{aligned}
$$

Since $h(x) \rightarrow-\infty$ and $g_{i}(x) \rightarrow-\infty$ as $x \rightarrow-\infty$, then it can be chosen a positive constant $B_{2}$ such that

$$
\dot{V}_{2} \leq-0.5 \text { for } x \leq-B_{2} \text {. }
$$

Then, we can conclude that there is a positive constant $\alpha_{2}$ such that the $x$-coordinate of the solutions of system (4) satisfies $x \geq-\alpha_{2}$ for $|y| \leq \beta$. On gathering the above whole discussion, one can see that the solutions of system (4) are ultimately bounded. Therefore, (3) has an $\omega$-periodic motion (solution). When $p(t)=K, K$-constant, (3) has a constant motion $x=c_{0}$. From (3), it can be seen that the constant $x=$ $c_{0}$ is given by $h\left(c_{0}\right)+g_{i}\left(c_{0}\right)=K$.

\section{Conflict of Interests}

The authors declare that there is no conflict of interests regarding the publication of this paper.

\section{References}

[1] R. E. Gaines and J. L. Mawhin, Coincidence Degree, and Nonlinear Differential Equations, vol. 568 of Lecture Notes in Mathematics, Springer, Berlin, Germany, 1977.

[2] T. Yoshizawa, "Stability theory by Liapunov's second method," Publications of the Mathematical Society of Japan 9, The Mathematical Society of Japan, Tokyo, Japan, 1966.

[3] J. M. Zhao, K. L. Huang, and Q. S. Lu, "The existence of periodic solutions for a class of functional-differential equations and their application," Applied Mathematics and Mechanics. English Edition, vol. 15, no. 1, pp. 49-59, 1994, translated from Applied Mathematics and Mechanics, vol. 15, no. 1, pp. 49-58, 1994. 
[4] T. A. Burton, Stability and Periodic Solutions of Ordinary and Functional Differential Equations, Dover, Mineola, NY, USA, 2005, Corrected Version of the 1985 Original.

[5] S. Ahmad and M. R. Rao, Theory of Ordinary differential Equations. With Applications in Biology and Engineering, Affiliated East-West Press, New Delhi, India, 1999.

[6] A. Constantin, "A note on a second-order nonlinear differential system," Glasgow Mathematical Journal, vol. 42, no. 2, pp. 195199, 2000.

[7] J. R. Graef, "On the generalized Liénard equation with negative damping," Journal of Differential Equations, vol. 12, pp. 34-62, 1972.

[8] L. H. Huang and J. S. Yu, "On boundedness of solutions of generalized Liénard's system and its application," Annals of Differential Equations, vol. 9, no. 3, pp. 311-318, 1993.

[9] Z. Jin, "Boundedness and convergence of solutions of a secondorder nonlinear differential system," Journal of Mathematical Analysis and Applications, vol. 256, no. 2, pp. 360-374, 2001.

[10] B. Liu and L. Huang, "Boundedness of solutions for a class of retarded Liénard equation," Journal of Mathematical Analysis and Applications, vol. 286, no. 2, pp. 422-434, 2003.

[11] J. E. Nápoles Valdés, "Boundedness and global asymptotic stability of the forced Liénard equation," Revista de la Unión Matemática Argentina, vol. 41, no. 4, pp. 47-59, 2000.

[12] C. X. Qian, "Boundedness and asymptotic behaviour of solutions of a second-order nonlinear system," The Bulletin of the London Mathematical Society, vol. 24, no. 3, pp. 281-288, 1992.

[13] C. Tunç, "A note on the bounded solutions," Applied Mathematics and Information Sciences, vol. 8, no. 1, pp. 393-399, 2014.

[14] C. Tunç, "A note on boundedness of solutions to a class of nonautonomous differential equations of second order," Applicable Analysis and Discrete Mathematics, vol. 4, no. 2, pp. 361-372, 2010.

[15] C. Tunç, "Boundedness results for solutions of certain nonlinear differential equations of second order," Journal of the Indonesian Mathematical Society, vol. 16, no. 2, pp. 115-126, 2010.

[16] C. Tunç, "Uniformly stability and boundedness of solutions of second order nonlinear delay differential equations," Applied and Computational Mathematics, vol. 10, no. 3, pp. 449-462, 2011.

[17] C. Tunç, "On the boundedness of solutions of a non-autonomous differential equation of second order," Sarajevo Journal of Mathematics, vol. 7(19), no. 1, pp. 19-29, 2011.

[18] C. Tunç, "Stability and uniform boundedness results for nonautonomous Lienard-type equations with a variable deviating argument," Acta Mathematica Vietnamica, vol. 37, no. 3, pp. 311325,2012

[19] C. Tunç, "On the stability and boundedness of solutions of a class of nonautonomous differential equations of second order with multiple deviating arguments," Afrika Matematika, vol. 23, no. 2, pp. 249-259, 2012.

[20] C. Tunç, "Stability to vector Lienard equation with constant deviating argument," Nonlinear Dynamics, vol. 73, no. 3, pp. 1245-1251, 2013.

[21] C. Tunç, "New results on the existence of periodic solutions for Rayleigh equation with state-dependent delay," Journal of Mathematical and Fundamental Sciences, vol. 45, no. 2, pp. 154162, 2013.
[22] C. Tunç and E. Tunç, "On the asymptotic behavior of solutions of certain second-order differential equations," Journal of the Franklin Institute, vol. 344, no. 5, pp. 391-398, 2007.

[23] J. Zhou, "Necessary and sufficient conditions for boundedness and convergence of a second-order nonlinear differential system," Acta Mathematica Sinica. Chinese Series, vol. 43, no. 3, pp. 415-420, 2000. 


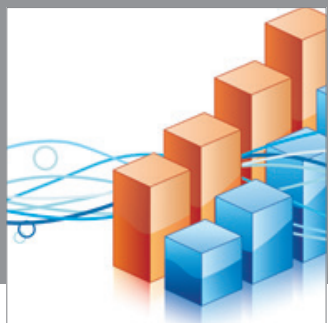

Advances in

Operations Research

mansans

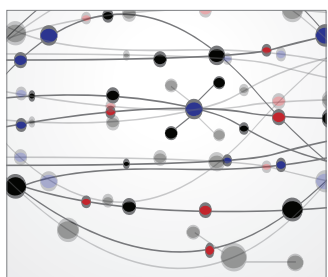

The Scientific World Journal
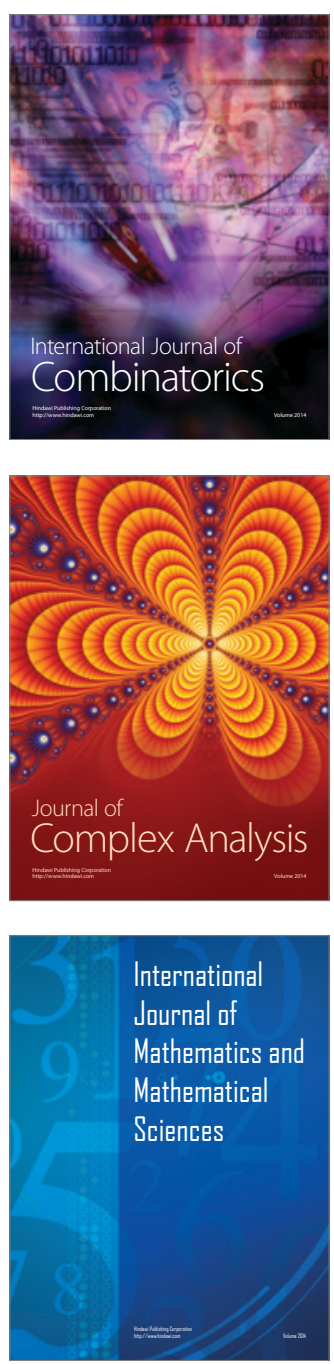
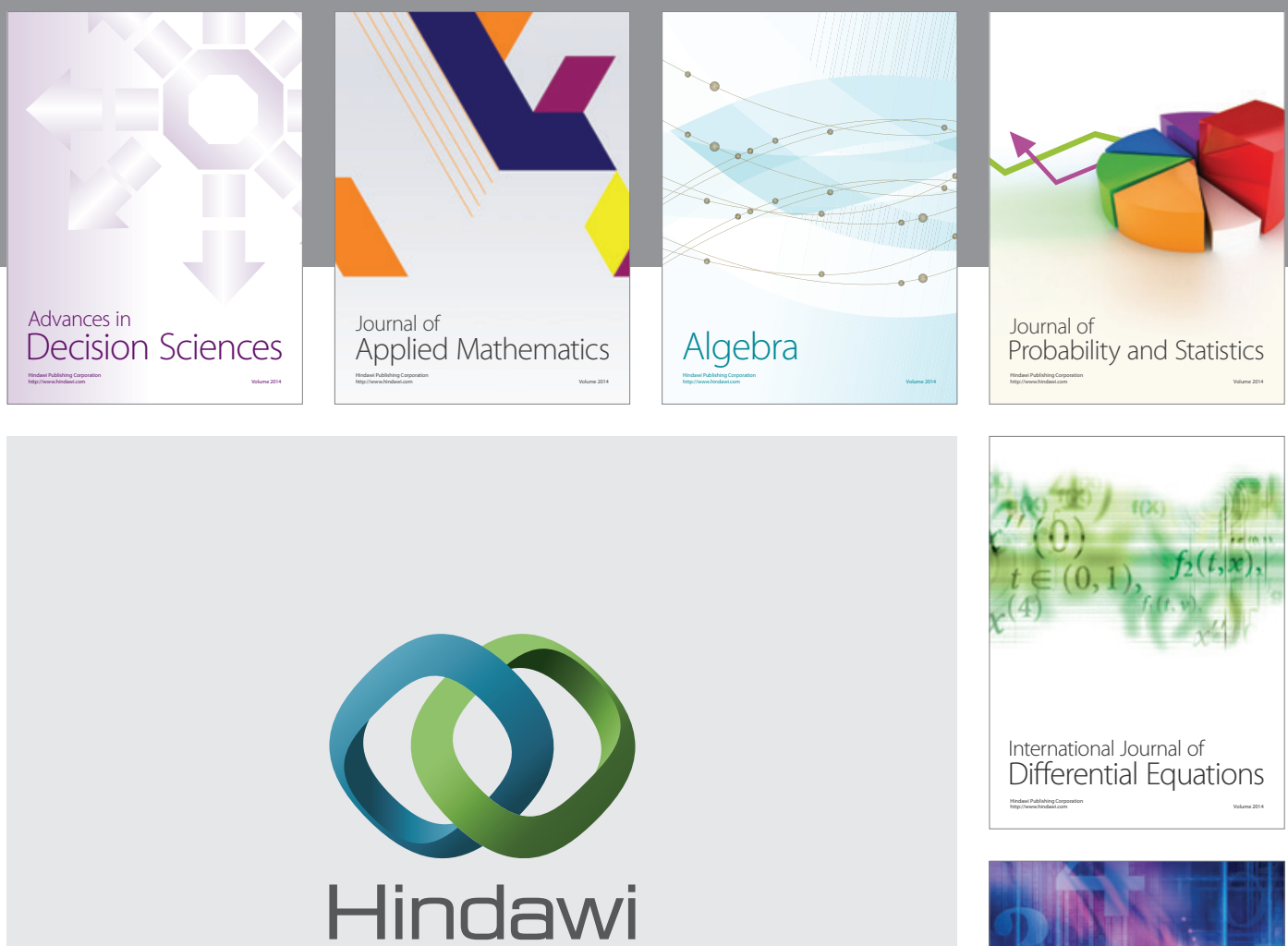

Submit your manuscripts at http://www.hindawi.com
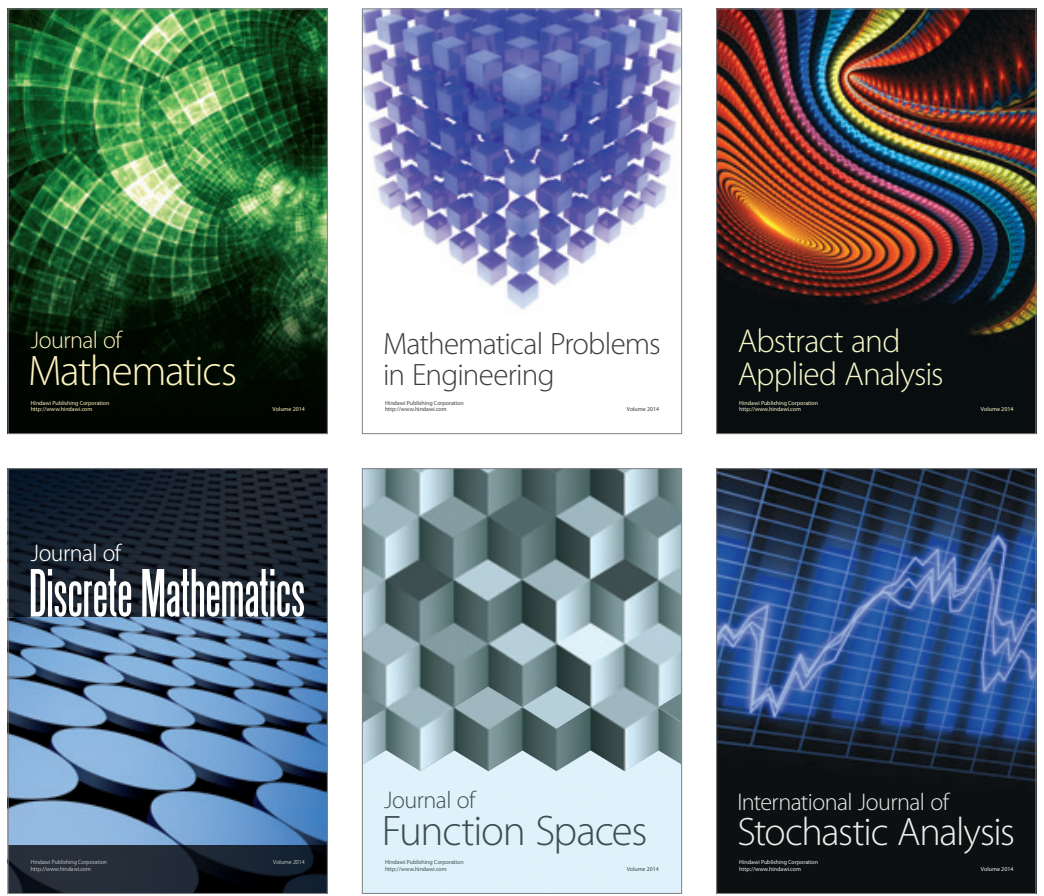

Journal of

Function Spaces

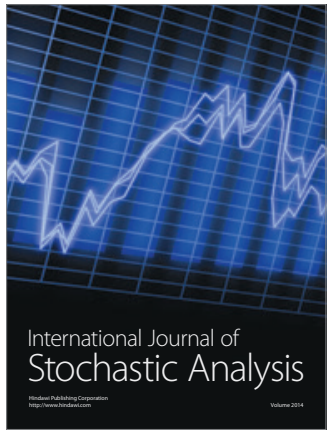

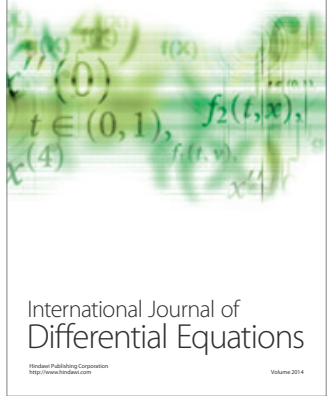
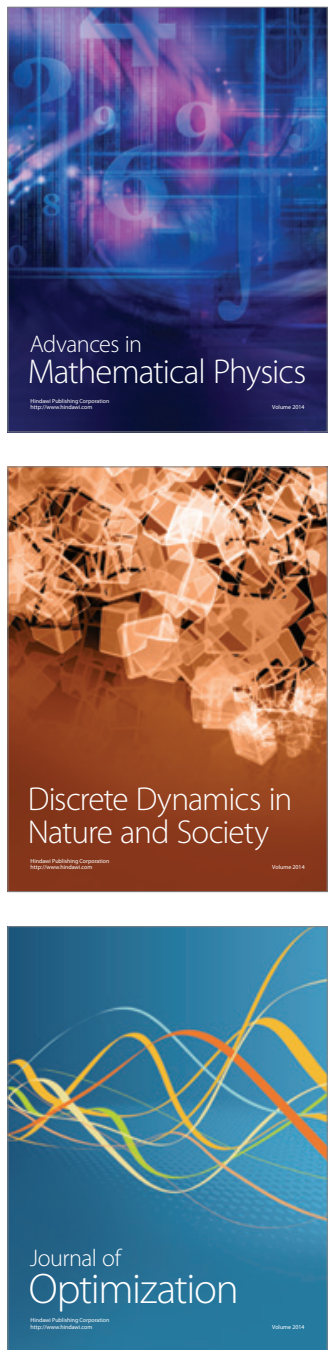